Globally, this survey will allow the WHO to provide a situational analysis of progress of current IPC and hand hygiene activities around the world and inform future efforts and resource use for IPC capacity building and improvement. Global surveys using the hand hygiene self-assessment framework were also conducted in 2011 and $2015,{ }^{3-5}$ making this year's survey even more crucial for tracking the implementation of hand hygiene and IPC on a global scale (Fig. 1).

Each improvement in IPC contributes toward quality UHC. "Clean care for all-it's in your hands!"

Acknowledgments. The authors alone are responsible for the views expressed in this article and they do not necessarily represent the views, decisions, or policies of the institutions with which they are affiliated. WHO takes no responsibility for the information provided or the views expressed in this paper.

Financial support. This work is supported by the World Health Organization (WHO), Geneva, Switzerland, and the Infection Control Program and WHO Collaborating Center on Patient Safety (SPCI/WCC), University of Geneva Hospitals and Faculty of Medicine, Geneva, Switzerland; hand hygiene research activities at the SPCI/WCC are also supported by the Swiss National Science Foundation (grant no. 32003B_163262).
Conflicts of interest. Didier Pittet works with WHO in the context of the WHO initiative "Private Organizations for Patient Safety-Hand Hygiene." All listed authors declare no financial support, grants, financial interests or consultancy that could lead to conflicts of interest.

\section{References}

1. What is universal coverage? World Health Organization website. http://www. who.int/health_financing/universal_coverage_definition/en/. Accessed February 19, 2019.

2. SAVE LIVES: Clean Your Hands. World Health Organization website. http://www.who.int/infection-prevention/campaigns/clean-hands/en/. Accessed February 19, 2019.

3. WHO Hand Hygiene Self-Assessment Global Survey for 2015. World Health Organization website. http://www.who.int/gpsc/5may/hhsa_framework2015/en/. Accessed February 19, 2019.

4. Allegranzi B, Conway L, Larson E, Pittet D. Status of the implementation of the World Health Organization multimodal hand hygiene strategy in United States of America health care facilities. Am J Infect Control 2014;42:224-230.

5. Kilpatrick C, Tartari E, Gayet-Ageron A, et al. Global hand hygiene improvement progress: two surveys using the WHO Hand Hygiene Self-Assessment Framework. J Hosp Infect 2018;100:202-206.

\title{
Real-world challenges in infection prevention: Differential implementation between stable and unstable patients may influence clinical effectiveness of interventions
}

\author{
Westyn Branch-Elliman MD, MMSc ${ }^{1,2,3}$ (1) and Marin Schweizer PhD 4 \\ ${ }^{1}$ VA Boston Healthcare System, Boston, Massachusetts, ${ }^{2}$ VA Center for Healthcare Organization and Implementation Research, Boston, Massachusetts, ${ }^{3} \mathrm{Harvard}$ \\ Medical School, Boston, Massachusetts and ${ }^{4}$ University of lowa and lowa City VA Health Care System, lowa City, lowa
}

To the Editor-We read "Implementation Strategies to Reduce Surgical Site Infections: A Systematic Review"1 by Ariyo et al with great interest. Identifying ways to improve implementation and uptake of infection prevention interventions is critical as the field moves toward translating and implementing evidence-based findings into day-to-day clinical practice.

A key finding of the systematic review by Ariyo et al was that few high-quality trials have examined different implementation strategies in infection prevention. Adding to the limitations of the current literature identified in the outstanding review, some of our recent work across multiple procedural and surgical specialties highlights the challenges in bringing infection prevention practices to the bedside and operating room. In particular, we found that implementation of prevention practices is unevenly applied across the spectrum of care. This variation in effective implementation may lead to significant bias and confounding that impacts the apparent benefits of different infection prevention interventions.

\footnotetext{
Author for correspondence: Westyn Branch-Elliman, Email: wbranche@bidmc.harvard. edu.

Cite this article: Branch-Elliman W and Schweizer M. (2019). Real-world challenges in infection prevention: Differential implementation between stable and unstable patients may influence clinical effectiveness of interventions. Infection Control \& Hospital Epidemiology, 40: 736-737, https://doi.org/10.1017/ice.2019.87
}

A consistent finding across multiple specialties and clinical care areas has been that more stable patients receive more systematic, protocolized care. Processes of care are standardized and systematically applied to the stable, elective patient population. However, that is not true for more urgent or emergent cases, which tend to be identified for surgical procedures in inpatient settings. This inherently sicker and higher-risk population may be less predictable and more difficult to track and control, with environmental barriers to implementation that do not exist for the outpatient population.

We have identified these findings across different types of invasive procedures and using different methodologies. For example, during qualitative interviews with frontline electrophysiologists, we learned that,

\footnotetext{
"[Cardiac device] patients come [to the electrophysiology laboratory] from a million different routes. They can be outpatients, they can be hospital to hospital transferred, they can be patients who present through the ER, they can come urgently from outpatient clinics. They can be transferred from another institution. [For all elected cases], ones who are scheduled [outpatients] get [chlorhexidine] at home, [the patients] do the cleaning process themselves... If the patient is [in the hospital], the nurses try to do the [chlorhexidine] on the day prior, but that is not uniform. For patients who are transferred from another hospital, they may have a temp wire and then go directly to the [electrophysiology] lab. From an infectious
} 
diseases perspective, the only thing that is standardized are the outpatients, and they are probably $50 \%$ of the volume."

This was also true for patients undergoing cardiac surgery in the STOP SSI study (cited by Ariyo et al) ${ }^{2}$ and similar subsequent unpublished studies. In the STOP SSI study, the significant reduction in complex $S$. aureus SSIs was only seen among orthopedic surgery patients but not cardiac surgery patients. The orthopedic surgery patients tended to be scheduled for elective surgery and had outpatient preoperative clinic visits in the 30 days prior to surgery. During this visit, the orthopedic patients were provided chlorhexidine body wash, were screened for $S$. aureus colonization and, if positive, were provided with a 5-day supply of mupirocin nasal ointment. In contrast, the cardiac surgery patients did not have a standardized outpatient preoperative clinic appointment and were often only seen in the inpatient setting.

Similarly, only $1.6 \%$ of patients undergoing urgent/emergent operations were fully adherent to the STOP SSI bundle, $40 \%$ of patients undergoing scheduled operations were fully adherent. This factor was reflected in the outcomes, in which there was only a statistically significant reduction in complex $S$. aureus SSIs among the scheduled operations and not the urgent/emergent operations. In a similar study performed in Veterans Affairs (VA) hospitals, a cardiac case manager from a small VA hospital stated:

"[The patient] could have a scheduled outpatient [cardiac catheterization] here and end up on a balloon pump, and then [be sent] via ambulance to the University for [urgent] open heart surgery. Those are not going to be ones we catch, obviously."

Many infection prevention interventions, such as chlorhexidine washes and MRSA nasal screening and decolonization, require significant upfront investment of time and resources. Thus, providing these services to less stable patients and to add-on patients is difficult, if not impossible. Environmental barriers do not allow for showering and time restrictions do not allow for MRSA screening and multiple-day decolonization regimens.

These implementation challenges are critical areas of future research. First, as a field, we need better ways to implement infection prevention interventions and to track which patients receive them and which do not. In addition to healthcareassociated infection outcomes, consideration of intermediate, implementation outcomes (eg, adherence to the intervention), should be included in assessments of efficacy and effectiveness, so that the potential for confounding by indication can be assessed and addressed. Second, we need to find better ways to triage infection prevention strategies. Under the current system, the most aggressive care is being targeted to the lowest-risk patients. Infection prevention interventions that can be rapidly implemented in the day-of-surgery area or the operating room could be more effective than interventions that need to be started multiple days before surgery. More research is needed to determine how we can improve implementation and overcome barriers for the most vulnerable patients, rather than targeting care where it is most convenient to do so.

Author ORCIDs. Westyn Branch-Elliman, Marin Schweizer, (iD) 0000-0002-3604-0093

0000-0002-9658-5124,

Acknowledgments. The authors would like to thank Heather Schacht Reisinger, Cassie Goedken, and Stacey Hockett-Sherlock for their work performing qualitative interviews.

Financial support. Funding for this letter was provided by the National Institutes of Health (grant no. NHLBI 1K12HL138049-01) and the Agency for Healthcare Research and Quality (AHRQ grant nos. HHSA2902006100021I, HS022467-02; VA HRS\&D CREATE CRE 12-291).

Conflicts of interest. M.S. received grant funding from PDI Healthcare.

\section{References}

1. Ariyo P, Zayed B, Riese V, et al. Implementation strategies to reduce surgical site infections: a systematic review. Infect Control Hosp Epidemiol 2019:1-14.

2. Schweizer ML, Chiang HY, Septimus E, et al. Association of a bundled intervention with surgical site infections among patients undergoing cardiac, hip, or knee surgery. JAMA 2015;313:2162-2171.

\title{
Rapid and economical detection of eight carbapenem-resistance genes in Enterobacteriaceae, Pseudomonas spp, and Acinetobacter spp directly from positive blood cultures using an internally controlled multiplex-PCR assay
}

\author{
Surojit Das MSc, $\mathrm{PhD}^{1}$, Subhanita Roy $\mathrm{MSc}^{1}$, Samadrita Roy $\mathrm{MSc}^{1}$, Gaurav Goel MD, $\mathrm{DNB}^{1}$, Kamini Walia PhD, MPH${ }^{2}$, \\ Sudipta Mukherjee MD, IDCCM, FNB, EDICM ${ }^{3}$, Sanjay Bhattacharya MD, DNB, FRCPath ${ }^{1}(\mathbb{0})$ and Mammen Chandy MD \\ FRCPA, FRACP, FRCP ${ }^{4}$
}

${ }^{1}$ Department of Microbiology, Tata Medical Center, Kolkata, India, ${ }^{2}$ Indian Council of Medical Research, New Delhi, India, ${ }^{3}$ Department of Critical Care, Tata Medical Center, Kolkata, India and ${ }^{4}$ Department of Clinical Hematology, Tata Medical Center, Kolkata, India

\footnotetext{
Author for correspondence: Dr Sanjay Bhattacharya, Emails: sanjay.bhattacharya@ tmckolkata.com or drsanjay1970@hotmail.com

Cite this article: Das S, et al. (2019). Rapid and economical detection of eight carbapenem-resistance genes in Enterobacteriaceae, Pseudomonas spp, and Acinetobacter spp directly from positive blood cultures using an internally controlled multiplex-PCR assay. Infection Control \& Hospital Epidemiology, 40: 737-739, https://doi.org/10.1017/ ice.2019.79
}

To the Editor-The World Health Organization (WHO) has recognized carbapenem-resistant Enterobacteriaceae (CRE), Pseudomonas aeruginosa (CRPsA), and Acinetobacter baumannii (CRAB) as critical pathogens that cause significant morbidity and mortality in patients with bloodstream infections (BSIs), 